International Journal of Pure and Applied Mathematics

Volume 98 No. 2 2015, 211-220

ISSN: 1311-8080 (printed version); ISSN: 1314-3395 (on-line version)

url: http://www.ijpam.eu

doi: http://dx.doi.org/10.12732/ijpam.v98i2.4

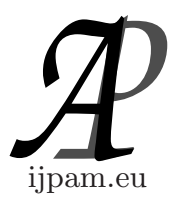

\title{
PRIME EXTENSION FILTRATION OF MODULES
}

\author{
T. Duraivel ${ }^{1 \S}$, S. Mangayarcarassy ${ }^{2}$, K. Premkumar ${ }^{3}$ \\ ${ }^{1}$ Department of Mathematics \\ Pondicherry University \\ Pondicherry, 605014, INDIA \\ ${ }^{2}$ Department of Mathematics \\ Pondicherry Engineering College \\ Pondicherry, 605014, INDIA \\ ${ }^{3}$ Department of Mathematics \\ Pondicherry University \\ Pondicherry, 605014, INDIA
}

\begin{abstract}
In this article we introduce a filtration for modules namely, regular prime extension filtration, which exist for all finitely generated modules over Noetherian rings. We show that any two such filtrations of a module have same length.
\end{abstract}

AMS Subject Classification: 13C05, 13E05, 13E15

Key Words: associated prime ideals, prime filtration, Noetherian ring, prime submodule and torsion free module

\section{Introduction}

Throughout this paper unless otherwise stated $A$ is a commutative Noetherian ring with identity and all modules are finitely generated and unitary. For standard reference and notations see [3] and [4].

Received: September 4, 2014

(C) 2015 Academic Publications, Ltd.

$\S$ Correspondence author url: www.acadpubl.eu 
It is well known that for a finitely generated module $M$ over a Noetherian ring $A$, there exists a filtration

$$
\mathcal{F}: 0=M_{0} \subset M_{1} \subset \cdots \subset M_{n-1} \subset M_{n}=M
$$

of submodules of $M$. The filtration $\mathcal{F}$ with the cyclic quotients $M_{i} / M_{i-1} \cong A / \mathrm{p}_{i}$ and $\mathrm{p}_{i} \in \operatorname{Supp}(M)$ is called a prime filtration of $M$. The set of prime ideals $\left\{\mathrm{p}_{1}, \ldots, \mathrm{p}_{n}\right\}$ which define the cyclic quotients of $\mathcal{F}$ will be denoted by $\operatorname{Supp}(\mathcal{F})$. We also have that $[4$, Theorem 6.5$]$

$$
\operatorname{Ass}(M) \subseteq \operatorname{Supp}(\mathcal{F}) \subset \operatorname{Supp}(M)
$$

In [2] Dress called the filtration $\mathcal{F}$ as weak prime decomposition of $M$, if for every $1 \leq i \leq n$, there exists a prime ideal $\mathrm{p}=\mathrm{p}_{i} \in \operatorname{Spec} A$ with $\mathrm{p}=\left(M_{i-1}: x\right)$ for all $x \in M_{i} \backslash M_{i-1}$. Clearly prime filtrations are weak prime decomposition and therefore every Noetherian $A$-module has a weak prime decomposition. For any Noetherian $A$-module $M$ always admits a weak prime decomposition $\mathcal{F}$ with $\operatorname{Supp}(\mathcal{F})=\operatorname{Ass}(M)$.

In this article for a proper submodule $N$ of $M$, we say a submodule $K$ of $M$ is prime extension of $N$ in $M$, if $N$ is a prime submodule of $K$. We say the filtration $\mathcal{F}$ is prime extension filtration of $M$ if $M_{i}$ is a prime extension of $M_{i-1}$ in $M$ for $1 \leq i \leq n$. It is clear that prime extension filtrations are nothing but weak prime decomposition of $M$. We call a prime extension filtration of $M$ is maximal prime extension (MPE) filtration if $M_{i}$ is maximal prime extension of $M_{i-1}$ for $1 \leq i \leq n$. We prove that if $\mathcal{F}$ is MPE filtration of $M$, then $\operatorname{Supp}(\mathcal{F})=\operatorname{Ass}(M)$.

Next we call an MPE filtration $N=M_{0} \subset M_{1} \subset \cdots \subset M_{n}=M$ with $\left(M_{i-1}: M_{i}\right)=\mathrm{p}_{i}$, as regular prime extension (RPE) filtration of $M$ over $N$, if $\mathrm{p}_{i}$ is a maximal element in Ass $\left(M / M_{i-1}\right)$. We prove that any two RPE filtration of $M$ over $N$ have same length. Hence this length is a numerical invariant for finitely generated modules over Noetherian ring.

\section{Prime Extension of Submodules}

Definition 1. Let $N$ be a proper submodule of $M$. A submodule $K$ of $M$ is called prime extension of $N$ in $M$, if $N$ is a prime submodule of $K$ and we denote it as $N \stackrel{\mathrm{p}}{\subset} K$, when $(N: K)=\mathrm{p}$.

Note 2. Let $M$ be an $A$-module and $N$ be a proper submodule of $M$. A submodule $K$ of $M$ is a p-prime extension of $N$ in $M$ if and only if $N \subsetneq K$ and $(N: x)=\mathrm{p}$, for every $x \in K \backslash N$. Moreover, $\operatorname{Ass}(K / N)=\{\mathrm{p}\}$. 
Lemma 3. Let $N$ be a proper submodule of an $A$-module $M$. Then $\mathrm{p} \in \operatorname{Ass}(M / N)$ if and only if there exists a p-prime extension of $N$ in $M$.

Proof. Let $\mathrm{p} \in \operatorname{Ass}(M / N)$, then $\mathrm{p}=\operatorname{ann}(x+N)$ for some $x \in M \backslash N$. Then $K=N+A x$ is a p-prime extension of $N$ in $M$.

Conversely, assume $K$ is a p-prime extension of $N$ in $M$. Then by note 2 , $\{\mathbf{p}\}=\operatorname{Ass}(K / N) \subset \operatorname{Ass}(M / N)$.

Lemma 4. Let $M$ be an A-module and $N$ be a proper submodule of $M$. If $M_{1}$ and $M_{2}$ are $\mathrm{p}_{1}$ and $\mathrm{p}_{2}$-prime extensions of $N$ respectively and $\mathrm{p}_{1} \neq \mathrm{p}_{2}$, then $M_{1} \cap M_{2}=N$.

Proof. Suppose $N \neq M_{1} \cap M_{2}$. Since $N$ is a $\mathrm{p}_{1}$-prime submodule of $M_{1}$, it is also $\mathrm{p}_{1}$-prime submodule of $M_{1} \cap M_{2}$ and hence $\left(N: M_{1} \cap M_{2}\right)=\mathrm{p}_{1}$. Similarly, since $N$ is a $\mathrm{p}_{2}$-prime submodule of $M_{2},\left(N: M_{1} \cap M_{2}\right)=\mathrm{p}_{2}$, a contradiction. Hence $M_{1} \cap M_{2}=N$.

Definition 5. Let $M$ be an $A$-module and $N$ be a proper submodule of $M$. A prime extension $K$ of $N$ is called maximal, if there is no prime extension $L$ of $N$ in $M$, which properly contains $K$.

Example 6. Let $M$ be a torsion free module over an integral domain $A$ and let $\mathrm{p}=(0)$. Then any nontrivial submodule of $M$ is a $\mathrm{p}$-prime extension of zero submodule in $M$. But the maximal p-prime extension of zero submodule in $M$ is $M$ itself.

Remark 7. Let $N$ be a proper submodule of $M$ and $\mathrm{p} \in \operatorname{Ass}(M / N)$. Since $M$ is Noetherian, there exists a maximal p-prime extension of $N$ in $M$.

Generally, for a proper submodule $N$ of $M$, Ass $(M / N)$ need not be contained in $\operatorname{Ass}(M)$. Next we show that $\operatorname{Ass}(M / N) \subseteq \operatorname{Ass}(M)$, if $N$ is a maximal prime extension of 0 in $M$. More generally, we prove the following theorem.

Theorem 8. Let $M$ be a finitely generated module over a Noetherian ring $A$ and $N$ be a proper submodule of $M$. If a submodule $K$ of $M$ is a maximal prime extension of $N$ in $M$, then $\operatorname{Ass}(M / K) \subseteq \operatorname{Ass}(M / N)$ and hence $\operatorname{Ass}(M / N)=\operatorname{Ass}(K / N) \cup \operatorname{Ass}(M / K)$.

Proof. Let $(N: K)=$ p. Suppose $\mathbf{q} \in \operatorname{Ass}(M / K)$ and $L$ be a $\mathbf{q}$ prime extension of $K$ in $M$.

Case(i) $\mathrm{p} \nsubseteq \nsubseteq \mathrm{q}$. For $x \in L \backslash K$ and $p \in \mathrm{p} \backslash \mathrm{q}$, we have that $p x \notin K$ and $(K: p x)=\mathrm{q}$. Also pq $x \subseteq N$ and therefore $\mathbf{q} \subseteq(N: p x)$. Clearly $(N: p x) \subset(K: p x)=\mathbf{q}$. Therefore $\mathbf{q}=(N: p x)$ and so $\mathbf{q} \in \operatorname{Ass}(M / N)$. 
Case(ii) $\mathrm{p} \subseteq \mathrm{q}$. Then $\mathrm{p} L \subseteq K$. Suppose $\mathrm{p} L \nsubseteq N$, then for some $x \in L \backslash K$ and $p \in \mathrm{p}, p x \notin N$. Clearly, $\mathrm{pq} x \subseteq N$ implies $\mathrm{q} \subseteq(N: p x)$. Since $K$ is a $\mathrm{p}$ prime extension of $N$ and $p x \in K$, then by note $2,(N: p x)=\mathrm{p} \subseteq \mathrm{q}$. Therefore $\mathbf{q} \in \operatorname{Ass}(M / N)$.

Next assume that $\mathrm{p} L \subseteq N$. Let $y \in L \backslash K$, then $\mathrm{p} \subseteq(N: y)$. We have that $(N: y) \subseteq(K: y)=\mathbf{q}$. Suppose $(N: y) \varsubsetneqq \mathbf{q}$, and let $q \in \mathbf{q} \backslash(N: y)$. But $q y \in K$ implies $(N: q y)=\mathrm{p}$, that is $(N: y) \subseteq \mathrm{p}$. This implies $(N: y)=\mathrm{p}$, for every $y \in L \backslash K$. Since $N \subset K$ is p-prime extension for every $y \in K \backslash N$, $(N: y)=\mathrm{p}$. Then for every $y \in L \backslash N,(N: y)=\mathrm{p}$ and so by note $2, L$ is a p-prime extension of $N$ in $M$, a contradiction to the maximality of $K$. Hence $(N: y)=\mathbf{q}$, and so $\mathbf{q} \in \operatorname{Ass}(M / N)$.

Taking $N=0$ in the above theorem we have the following corollary.

Corollary 9. If $K$ is a maximal prime extension of 0 in $M$, then $\operatorname{Ass}(M / K) \subset \operatorname{Ass}(M)$ and hence $\operatorname{Ass}(M)=\operatorname{Ass}(K) \cup \operatorname{Ass}(M / K)$.

Example 10. Maximal prime extension of a proper submodule of $M$ need not be unique. For example, let $k$ be a field and $x, y$ be indeterminates. Let $A=k[x, y]$ and $M=\frac{k[x, y]}{\left(x^{2}, x y\right)}$ and $X, Y$ be images of $x, y$ in $M$. Then $\mathrm{p}=(x)$ belongs to Ass $M$, but $A Y, A(X+Y)$ are two different maximal $\mathrm{p}$ prime extension of 0 in $M$.

Next we show that the maximal p-prime extension of a submodule $N$ is unique, if $\mathrm{p}$ is a maximal element in $\operatorname{Ass}(M / N)$.

Theorem 11. Let $M$ be an A-module, $N$ be a proper submodule of $M$ and $\mathrm{p}$ be a maximal element in $\operatorname{Ass}(M / N)$. Then

$$
L=\{x \in M \mid(N: x) \supseteq \mathrm{p}\}
$$

is a unique maximal p-prime extension of $N$ in $M$.

Proof. Clearly, $L$ is a submodule of $M$. Since $\mathrm{p} \in \operatorname{Ass}(M / N), \mathrm{p}=(N: x)$ for some $x \in M \backslash N$. By assumption $x \in L$ and so $N$ is properly contained in $L$. Now consider the nonempty set,

$$
\Sigma=\{(N: z) \mid z \in L \backslash N\}
$$

We have that, $\mathrm{p} \subseteq(N: z)$ for every $(N: z) \in \Sigma$. Since $A$ is Noetherian, $\Sigma$ has a maximal element, say $(N: y)$. Then clearly $(N: y)$ is a prime ideal and so $(N: y) \in \operatorname{Ass}(M / N)$. Since $(N: y) \supseteq \mathrm{p}$ and $\mathrm{p}$ is a maximal element in 
$\operatorname{Ass}(M / N),(N: y)=\mathrm{p}$. Therefore $\Sigma=\{\mathbf{p}\}$ and hence for every $x \in L \backslash N$, $(N: x)=\mathrm{p}$, then by note $2, L$ is a $\mathrm{p}$-prime extension of $N$ in $M$.

Let $K$ be any p-prime extension of $N$ in $M$, then by note $2,(N: x)=\mathrm{p}$, for every $x \in K \backslash N$ and by definition of $L, x \in L$. Hence $K \subseteq L$ and so $L$ is unique maximal p-prime extension of $N$ in $M$.

\section{Maximal Prime Extension Filtration}

Next we define prime extension filtration of a module and we show that for every finitely generated module over a Noetherian ring prime extension filtration of $M$ over $N$ exists.

Definition 12. Let $M$ be an $A$-module. A filtration of submodules of $M$, $N=M_{0} \subset M_{1} \subset \cdots \subset M_{n}=M$ is called prime extension filtration of $M$ over $N$, if $M_{i}$ is a prime extension of $M_{i-1}$ in $M$, for $1 \leq i \leq n$. We say a prime ideal p occurs in this prime extension filtration, if $M_{i}$ is a maximal p-prime extension of $M_{i-1}$ for some $i$.

We may also write the filtration as $N=M_{0} \stackrel{\mathrm{p}_{1}}{\subset} M_{1} \subset \cdots \subset M_{n-1} \stackrel{\mathrm{p}_{n}}{\subset} M_{n}=M$, where $M_{i}$ is $\mathrm{p}_{i}$-prime extension of $M_{i-1}$.

If $N=0$, then we say it as prime extension filtration of $M$.

For a proper submodule $N$ of $M$, there exist a prime extension filtration of finite length of $M$ over $N$.

Definition 13. A prime extension filtration of $M$ over $N$ is called maximal prime extension (MPE) filtration of $M$ over $N$, if each prime extension in the filtration is maximal. If $N=0$ then we call it as MPE filtration of $M$.

For example $0 \stackrel{(0)}{\subset} 2 \mathbb{Z} \stackrel{(2)}{\subset} \mathbb{Z}$ is a prime extension filtration. But the only maximal prime extension filtration of $\mathbb{Z}$ is $0 \stackrel{(0)}{\subset} \mathbb{Z}$.

Note that by remark 7, each finitely generated module over a Noetherian ring has a MPE filtration of finite length.

Dress [2, PD7] proved that each finitely generated $A$-module $M$ admits a filtration $0=M_{0} \subset M_{1} \subset \cdots \subset M_{n}=M$, where each $M_{i}$ is $\mathrm{p}_{i}$-prime extension of $M_{i-1}$ such that $\operatorname{Ass}(M)=\left\{\mathrm{p}_{1}, \ldots, \mathrm{p}_{n}\right\}$. Now we show that the MPE filtrations of $M$ are such filtration for $M$.

Proposition 14. Let $N$ be the proper submodule of $M$. If

$$
N=M_{0} \subset M_{1} \subset \cdots \subset M_{n}=M
$$


is a MPE filtration of $M$ over $N$, with $\left(M_{i-1}: M_{i}\right)=\mathrm{p}_{i}$ for every $i$, then $\operatorname{Ass}\left(M / M_{i}\right) \subseteq \operatorname{Ass}(M / N)$, for $1 \leq i \leq n$, and $\operatorname{Ass}(M / N)=\left\{\mathrm{p}_{1}, \ldots, \mathrm{p}_{n}\right\}$.

Proof. If $n=1$, the result is trivial. So we assume $n>1$ and prove by induction on $i$. If $i=1$, by theorem $8, \operatorname{Ass}\left(M / M_{1}\right) \subseteq \operatorname{Ass}(M / N)$. Now we assume $\operatorname{Ass}\left(M / M_{i-1}\right) \subseteq \operatorname{Ass}(M / N)$. Since $M_{i}$ is a maximal $\mathrm{p}_{i}$-prime extension of $M_{i-1}$ in $M$, by theorem $8, \operatorname{Ass}\left(M / M_{i}\right) \subseteq \operatorname{Ass}\left(M / M_{i-1}\right)$. Therefore $\operatorname{Ass}\left(M / M_{i}\right) \subseteq \operatorname{Ass}(M / N)$.

We prove the other part by induction on length of MPE filtration. If $n=1$ then by note $2, \operatorname{Ass}(M / N)=\left\{p_{1}\right\}$. Now we assume this result is true for the MPE filtration of length less than $n$. Considering $M_{n-1} / N$ as a submodule of $M / N$, we have that $\operatorname{Ass}(M / N) \subseteq \operatorname{Ass}\left(M_{n-1} / N\right) \cup \operatorname{Ass}\left(M / M_{n-1}\right)$. Now by previous paragraph $\operatorname{Ass}\left(M / M_{n-1}\right) \subseteq \operatorname{Ass}(M / N)$, that is $\operatorname{Ass}(M / N)=$ $\operatorname{Ass}\left(M_{n-1} / N\right) \cup \operatorname{Ass}\left(M / M_{n-1}\right)$. Since $M$ is a $\mathrm{p}_{n}$-prime extension of $M_{n-1}$, by note $2, \operatorname{Ass}\left(M / M_{n-1}\right)=\left\{\mathrm{p}_{n}\right\}$. Also

$$
N=M_{0} \stackrel{p_{1}}{\subset} M_{1} \subset \cdots \stackrel{p_{n-1}}{\subset} M_{n-1}
$$

is a MPE filtration of $M_{n-1}$ over $N$ of length $n-1$. Then by assumption $\operatorname{Ass}\left(M_{n-1} / N\right)=\left\{\mathbf{p}_{1}, \ldots, \mathbf{p}_{n-1}\right\}$, and so $\operatorname{Ass}(M / N)=\left\{\mathbf{p}_{1}, \ldots, \mathbf{p}_{n}\right\}$.

If we take $N=0$ in proposition 14, then we have the following corollary.

Corollary 15. Let $M$ be an A-module and

$$
0=M_{0} \stackrel{p_{1}}{\subset} M_{1} \subset \cdots \stackrel{p_{n}}{\subset} M_{n}=M
$$

be a $M P E$ filtration of $M$. Then $\operatorname{Ass}(M)=\left\{\mathrm{p}_{1}, \ldots, \mathrm{p}_{n}\right\}$. In other words a prime ideal $\mathrm{p}$ occurs in a MPE filtration of $M$ if and only if $\mathrm{p} \in \operatorname{Ass}(M)$.

Note, the above result is not true for prime extension filtration which are not maximal. For example we have the prime extension filtration $0 \stackrel{(0)}{\subset} 2 \mathbb{Z} \stackrel{(2)}{\subset} \mathbb{Z}$, but the prime ideal $(2) \notin$ Ass $\mathbb{Z}$.

Proposition 16. Let $N$ be a proper submodule of $M$ and let $\mathrm{p}_{1}$ and $\mathrm{p}_{2}$ be maximal element in $\operatorname{Ass}(M / N)$ and $N \stackrel{\mathrm{p}_{1}}{\subset} K_{1} \stackrel{\mathrm{p}_{2}}{\subset} L$ be a filtration, such that $N \subset K_{1}$ is maximal $\mathrm{p}_{1}$-prime extension in $M$ and $K_{1} \subset L$ is maximal $\mathrm{p}_{2}$-prime extension in $M$. Then there exist a submodule $K_{2}$ of $M$ such that $N \stackrel{p_{2}}{\subset} K_{2} \stackrel{p_{1}}{\subset} L$ is a filtration, with $N \subset K_{2}$ is maximal $p_{2}$-prime extension in $M$ and $K_{2} \subset L$ is maximal $\mathrm{p}_{1}$-prime extension in $M$. 
Proof. Since $\mathrm{p}_{2}$ is a maximal element in $\operatorname{Ass}(M / N)$, by theorem 11, there exist a unique maximal $\mathrm{p}_{2}$-prime extension $K_{2}$ of $N$ in $M$. We have to show that $L$ is maximal $\mathrm{p}_{1}$-prime extension of $K_{2}$ in $M$.

Let $a \in\left(K_{2}: L\right)$, then $a L \subseteq K_{2}$. Now $a K_{1} \subseteq a L \subseteq K_{2}$ and $a K_{1} \subseteq K_{2} \cap K_{1}$, then by lemma $4, K_{2} \cap K_{1}=N$. Therefore $a K_{1} \subseteq N$, implies $a \in\left(N: K_{1}\right)=\mathrm{p}_{1}$. So $\left(K_{2}: L\right) \subseteq \mathrm{p}_{1}$. Let $a \in \mathrm{p}_{1}$. Suppose $a x \notin K_{2}$, for some $x \in L$. Since $K_{2}$ is the unique maximal $\mathrm{p}_{2}$-prime extension of $N$ in $M$, by theorem 11, $\mathrm{p}_{2} \nsubseteq(N: a x)$. That is there exist an element $p_{2} \in \mathrm{p}_{2}$, such that $p_{2}(a x) \notin N$ and therefore $p_{2} x \notin N$ and $a \notin\left(N: p_{2} x\right)$. Since $x \in L$ and $L$ is $p_{2}$-prime extension of $K_{1}$, $p_{2} x \in K_{1}$ and hence $\left(N: p_{2} x\right)=\mathrm{p}_{1}$. This implies $a \notin \mathrm{p}_{1}$, a contradiction. So $a x \in K_{2}$, for every $x \in L$, that is $a \in\left(K_{2}: L\right)$ and therefore $\mathrm{p}_{1} \subseteq\left(K_{2}: L\right)$. So we proved $\mathrm{p}_{1}=\left(K_{2}: L\right)$.

Let $x \in K_{2}$. Since $N \subset K_{2}$ is maximal $\mathrm{p}_{2}$-prime extension by theorem 11 , $(N: x) \supseteq \mathrm{p}_{2}$. That is $\mathrm{p}_{2} x \subseteq N \subset K_{1}$ and this implies $\mathrm{p}_{2} \subseteq\left(K_{1}: x\right)$. Again by theorem 11, $x \in L$, so $K_{2} \subseteq L$. Since $\left(K_{2}: L\right)=\mathrm{p}_{1}, K_{2}$ is proper submodule of $L$.

Let $a x \in K_{2}$ for some $x \in L$ and $a \in A$. Suppose $x \notin K_{2}$, then by theorem $11, \mathrm{p}_{2} \nsubseteq(N: x)$. That is there exist an element $p_{2} \in \mathrm{p}_{2}$ such that $p_{2} x \notin N$. But $a x \in K_{2}$, implies $p_{2} a x \in N$. Since $p_{2} x \in K_{1}, a \in\left(N: p_{2} x\right)=\mathrm{p}_{1}$. This implies that $L$ is a $\mathrm{p}_{1}$-prime extension of $K_{2}$.

Next we prove $L$ is maximal $\mathrm{p}_{1}$-prime extension of $K_{2}$ in $M$. Since $K_{2}$ is maximal prime extension of $N$, and by theorem $8, \operatorname{Ass}\left(M / K_{2}\right) \subset \operatorname{Ass}(M / N)$, so $\mathrm{p}_{1}$ is a maximal element in $\operatorname{Ass}\left(M / K_{2}\right)$. Then by theorem 11, there exist a unique maximal $\mathrm{p}_{1}$-prime extension $M^{\prime}$ of $K_{2}$ in $M$. So it is enough to show $M^{\prime} \subseteq L$.

Let $x \in M^{\prime}$, then $\mathrm{p}_{1} \subseteq\left(K_{2}: x\right)$. That is for every $p_{1} \in \mathrm{p}_{1}, p_{1} x \in K_{2}$ and again by theorem 11, on maximal $\mathrm{p}_{2}$-prime extension $N \subset K_{2}, \mathrm{p}_{2} \subseteq\left(N: p_{1} x\right)$. Therefore $p_{1} p_{2} x \in N$ for every $p_{2} \in \mathrm{p}_{2}$. In other words $\mathrm{p}_{1} \subseteq\left(N: p_{2} x\right)$, then by $N \subset K_{1}$ is $\mathrm{p}_{1}$-maximal prime extension, $p_{2} x \in K_{1}$ for every $p_{2} \in \mathrm{p}_{2}$, which implies $\mathrm{p}_{2} \subseteq\left(K_{1}: x\right)$. Since $K_{1} \subset L$ is maximal $\mathrm{p}_{2}$-prime extension, we have that $x \in L$. So $M^{\prime} \subseteq L$. Hence $L$ is a maximal $\mathrm{p}_{1}$-prime extension of $K_{2}$ in $M$. This completes the proof.

Next we show that we can modify an MPE filtration of $M$ over $N$ without altering its length so that the occurrences of any two prime ideals $\mathbf{p}_{i}$ and $\mathbf{p}_{i+1}$ with $\mathrm{p}_{i} \nsubseteq \mathrm{p}_{i+1}$ and $\mathrm{p}_{i+1} \nsubseteq \mathrm{p}_{i}$ can be interchanged.

Corollary 17. Let $N$ be any proper submodule of an $A$-module $M$. If $N=M_{0} \subset \cdots \subset M_{i-1} \stackrel{p_{i}}{\subset} M_{i} \stackrel{p_{i+1}}{\subset} M_{i+1} \subset \cdots \subset M_{n}=M$ is a MPE filtration of $M$ over $N$, with $\mathbf{p}_{i}$ and $\mathrm{p}_{i+1}$ are distinct maximal elements in $\operatorname{Ass}\left(M / M_{i-1}\right)$, 
then there exist a submodule $K_{i}$ of $M$, such that $N=M_{0} \subset \cdots \subset M_{i-1} \stackrel{p_{i+1}}{\subset}$ $K_{i} \stackrel{\mathrm{p}_{i}}{\subset} M_{i+1} \subset \cdots M_{n}=M$ is a MPE filtration of $M$ over $N$.

Proof. Take $N=M_{i-1}, K_{1}=M_{i}$ and $L=M_{i+1}$ in proposition 16 .

Example 18. Generally two MPE filtration of an $A$-module need not have same length. Consider the module $M$ given in example 10. Then we have two MPE filtration

$$
0 \stackrel{(x, y)}{\complement} A X \stackrel{(x)}{\complement} M \text { and } 0 \stackrel{(x)}{\complement} A Y \stackrel{(x, y)}{\complement} A X+A Y \stackrel{(x, y)}{\subset} M
$$

of $M$ with length 2 and 3 respectively.

\section{Regular Prime Extension Filtration}

Now we give the following definition.

Definition 19. Let $N$ be a proper submodule of an $A$-module $M$. An MPE filtration $N=M_{0} \subset M_{1} \subset \cdots \subset M_{n}=M$ of $M$ over $N$ is called Regular Prime Extension (RPE) filtration of $M$ over $N$, if $\mathrm{p}_{i}$ is maximal element in $\operatorname{Ass}\left(M / M_{i-1}\right)$, for $1 \leq i \leq n$. If $N=0$, then we call it as an RPE filtration of $M$.

In this section we will show all RPE filtration of $M$ over $N$ have unique length and for any prime ideal $\mathrm{p}$ of $A$, the number of times $\mathrm{p}$ occurs on any two RPE filtration are same.

Lemma 20. Let $M$ be an $A$-module and $N$ be the proper submodule of M. If

$$
N=M_{0} \stackrel{\mathrm{p}_{1}}{\subset} M_{1} \subset \cdots \subset M_{i-1} \stackrel{\mathrm{p}_{i}}{\subset} M_{i} \stackrel{\mathrm{p}_{i+1}}{\subset} M_{i+1} \subset \cdots \subset M_{n}=M
$$

is a RPE filtration of $M$ over $N$, and if $\mathrm{p}_{i+1} \nsubseteq \mathrm{p}_{i}$ for some $i$, then there exist a submodule $K_{i}$ of $M$, such that

$$
N=M_{0} \stackrel{p_{1}}{\subset} M_{1} \subset \cdots \stackrel{p_{i-1}}{\subset} M_{i-1} \stackrel{p_{i+1}}{\subset} K_{i} \stackrel{p_{i}}{\subset} M_{i+1} \stackrel{p_{i+2}}{\subset} \cdots \subset M_{n}=M
$$

is a RPE filtration of $M$ over $N$.

Proof. Since $\mathrm{p}_{i} \nsubseteq \mathrm{p}_{i+1}$ and by assumption $\mathrm{p}_{i+1} \nsubseteq \mathrm{p}_{i}$ also $M_{i+1}$ is maximal $\mathrm{p}_{i+1}$-prime extension of $M_{i}, \mathrm{p}_{i+1}$ is maximal element in Ass $\left(M / M_{i}\right)$. This 
implies $\mathrm{p}_{i+1}$ is maximal element in Ass $\left(M / M_{i}\right) \cup\left\{\mathrm{p}_{i}\right\}=$ Ass $\left(M / M_{i-1}\right)$. So both $\mathrm{p}_{i}$ and $\mathrm{p}_{i+1}$ are distinct maximal elements in $\operatorname{Ass}\left(M / M_{i-1}\right)$. Then by corollary 17 , there exist a prime extension $K_{i}$ of $M_{i-1}$ such that (2) is MPE filtration of $M$ over $N$. Since $\mathrm{p}_{i+1}$ is maximal element in Ass $\left(M / M_{i-1}\right),(2)$ is RPE filtration of $M$ over $N$.

Corollary 21. Let $N$ be a proper submodule of an $A$-module $M$ and $\mathrm{p}$ be a maximal element in $\operatorname{Ass}(M / N)$. If there exist an $R P E$ filtration of $M$ over $N$ with length $n$, then there exist an RPE filtration of $M$ over $N$ with length $n$, such that $\mathbf{p}$ occurs in the first place and for each prime ideal $\mathbf{q}$ the number of times q occurs in these two filtration are equal.

Proof. Suppose

$$
N=M_{0} \stackrel{p_{1}}{\subset} M_{1} \subset \cdots \stackrel{p_{i-1}}{\subset} M_{i-1} \stackrel{p}{\subset} M_{i} \stackrel{p_{i+1}}{\subset} M_{i+1} \subset \cdots \stackrel{p_{n}}{\subset} M_{n}=M
$$

is a RPE filtration of $M$ over $N$, with $i$ be the least integer such that $\mathbf{p}=\mathrm{p}_{i}$. Since $\mathrm{p}$ is a maximal element in $\operatorname{Ass}(M / N), \mathrm{p} \not \subset \mathrm{p}_{j}$ for $1 \leq j \leq n$ and in particular $\mathrm{p} \not \subset \mathrm{p}_{i-1}$. Then by lemma 20, there exist a submodule $K_{i-1}$ of $M$, such that $N=M_{0} \stackrel{\mathrm{p}_{1}}{\subset} M_{1} \subset \cdots \subset M_{i-2} \stackrel{\mathrm{p}}{\subset} K_{i-1} \stackrel{p_{i-1}}{\subset} M_{i} \subset \cdots \stackrel{\mathrm{p}_{n}}{\subset} M_{n}=M$ is a RPE filtration of $M$ over $N$. Continuing the process, finally we will have a filtration

$$
N=M_{0} \stackrel{p}{\subset} K_{1} \stackrel{p_{1}}{\subset} \ldots \stackrel{p_{i-2}}{\subset} K_{i-1} \stackrel{p_{i-1}}{\complement} M_{i} \stackrel{p_{i+1}}{\subset} M_{i+1} \subset \cdots \stackrel{p_{n}}{\subset} M_{n}=M
$$

is also a RPE filtration of $M$ over $N$. Obviously, for any prime ideal q, the number of times q occurs in (3) and (4) are same.

Next we show that any two RPE filtration of $M$ over $N$ have same length.

Theorem 22. Let $N$ be a proper submodule of $M$. For any prime ideal $\mathrm{p}$ of $A$ the number of times $\mathbf{p}$ occurs in any two RPE filtration of $M$ over $N$ are equal, and hence any two RPE filtration of $M$ over $N$ have same length.

Proof. Let

$$
\begin{aligned}
& N=M_{0} \stackrel{p_{1}}{\subset} M_{1} \subset \cdots \subset M_{n-1} \stackrel{p_{n}}{\subset} M_{n}=M \\
& N=K_{0} \stackrel{\mathrm{q}_{1}}{\subset} K_{1} \subset \cdots \subset K_{m-1} \stackrel{\mathrm{q}_{m}}{\subset} K_{m}=M
\end{aligned}
$$

be the two RPE filtration of $M$ over $N$ with length $n$ and $m$ respectively. We prove this result by induction on $n$. 
If $n=1$, then it is trivial. We assume that the result is true, if the length of the RPE filtration (5) is less than $n$. Now $\mathrm{p}_{1} \in \operatorname{Ass}(M / N)=\left\{\mathrm{q}_{1}, \ldots, \mathrm{q}_{m}\right\}$, implies $\mathrm{p}_{1}=\mathrm{q}_{i}$ for some $i$. Since $\mathrm{p}_{1}$ is maximal element in Ass $(M / N)$, then by corollary 21 , we can assume without loss of generality $p_{1}=q_{1}$. Then by theorem $11, K_{1}=M_{1}$ and we have a RPE filtration

$$
\begin{gathered}
M_{1}=M_{1} \subset M_{2} \subset \cdots \subset M_{n-1} \subset M_{n}=M \\
\text { and } \\
M_{1}=K_{1} \subset K_{2} \subset \cdots \subset K_{m-1} \subset K_{m}=M
\end{gathered}
$$

of $M$ over $M_{1}$. Then by assumption for any prime ideal $\mathrm{p}$ the number of times $\mathrm{p}$ occurs in the RPE filtration (7) and (8) are same. So the number of times p occurs in the RPE filtration (5) and (6) are same. And so any two RPE filtration of $M$ over $N$ have same length.

\section{Acknowledgments}

The third author was financially supported by SRF of CSIR, India.

\section{References}

[1] N. Bourbaki, Commutative algebra, Herman, Paris (1967).

[2] A. Dress, A new algebraic criterion for shellability, Beitrage zur Alg. und Geom. 34(1) (1993), 45-55.

[3] D. Eisenbud, Commutative algebra, with a view toward algebraic geometry, Springer, New York (1995).

[4] H. Matsumura, Commutative Ring Theory, Cambridge university press, (1989). 